\title{
Thermal Water from Uriage-les-Bains Exerts DNA Protection, Induction of Catalase Activity and Claudin-6 Expression on UV Irradiated Human Skin in Addition to Its Own Antioxidant Properties
}

\author{
Francine Joly ${ }^{1}$, Jean-Eric Branka ${ }^{2}$, Luc Lefeuvre ${ }^{3}$ \\ ${ }^{1}$ Laboratoire SEPhRAPHARMA, Puteaux, France \\ ${ }^{2}$ EPHYSCIENCE, Nantes, France \\ ${ }^{3}$ Laboratoires Dermatologiques d'Uriage, Neuilly sur Seine, France \\ Email: francine-joly@sephrapharma.com
}

Received 4 February 2014; revised 1 March 2014; accepted 8 March 2014

Copyright (C) 2014 by authors and Scientific Research Publishing Inc.

This work is licensed under the Creative Commons Attribution International License (CC BY).

http://creativecommons.org/licenses/by/4.0/

(c) (i) Open Access

\begin{abstract}
Aim: In order to decipher the mechanisms underlying the known protective effects of the thermal water from Uriage-les-Bains (TWFULB) on the skin barrier function, we studied its antioxidant properties as well as its effect on the expression of the tight-junctional protein claudin-6, a candidate tumor suppressor factor. Study Design/Methods: In a first step, TBARS and SOD activity assays were performed in an in vitro model of human dermal fibroblasts treated by hypoxanthine/xanthine oxidase (HO/XO) mixture, in order to evaluate the own antioxidant effect of the thermal water. In a second step, human keratinocytes irradiated or not by UVB were used to evaluate the protective role of TWFULB on nuclear DNA damage using a comet assay. In a third step, an ex vivo model of human skin explants irradiated or not by UVA and UVB was used to evaluate the effect of TWFULB on the intracellular catalase activity and on the cutaneous claudin- 6 expression. Results: TWFULB showed significant protective effects against oxidative stress induced by HO/XO: the cell viability was improved and the lipid peroxidation was reduced. The tested thermal water also showed significant SOD-like activity and protective effect on the UVB-stressed DNA. Considering the ex vivo models of skin explants, TWFULB was able to counterbalance the "negative" effect of UVB on the intracellular catalase activity and on the cutaneous claudin-6 expression. Conclusion: This multiparametric approach shows the antioxidant activity of TWFULB and emphasizes its role in the DNA protection of the cutaneous tissue in front of the UV irradiations, and finally suggests that some effects could involve the candidate suppressor functions of claudin- 6 .
\end{abstract}

\footnotetext{
*Corresponding author.
}

How to cite this paper: Joly, F., Branka, J.-E. and Lefeuvre, L. (2014) Thermal Water from Uriage-les-Bains Exerts DNA Protection, Induction of Catalase Activity and Claudin-6 Expression on UV Irradiated Human Skin in Addition to Its Own Antioxidant Properties. Journal of Cosmetics, Dermatological Sciences and Applications, 4, 99-106. http://dx.doi.org/10.4236/jcdsa.2014.42015 
Keywords

Human Skin; UVA-UVB; Thermal Water from Uriage-les-Bains; Claudin-6; Antioxidant Effect; SOD-Like Effect; Catalase

\section{Introduction}

Numerous cosmetic products contain thermal spring waters coming from different origins and showing different physiological effects including in relation to their ionic compositions. Sulphurous thermal waters for example, have shown antioxidant properties [1]-[3], DNA protective effects [1] and inhibitory activities on neutrophil elastase [2]. Other thermal waters, such as Avene thermal water, also showed antioxidant, anti-inflammatory and immunomodulating properties and were moreover able to inhibit the TNF $\alpha$ induced-adhesion molecules in human endothelial cells [4] [5]. "La Roche Posay" thermal water for its part is rich in selenium, and displays antioxidant, anti-inflammatory, immunodulating and anti-irritant potential [6]. Another example coming from Korea also clarified the action of the spa spring water from "Yong-gung oncheon" on cytokine expression in human keratinocyte HaCaT cells and on differentiation of CD4+ T Cells [7].

Of course, protecting skin from oxidation, inflammatory processes etc., is very important for this tissue which constitutes the first line of defense of the organism. However, in addition to these considerations, the evaluation of the potential activity of a thermal water in preventing the nuclear DNA damages induced by UV irradiations in cutaneous tissue, and on the subsequent consequences is a crucial, and yet relatively unexplored issue.

In a recent article, we demonstrated the beneficial effect of the thermal water from Uriage-les-Bains (TWFULB) on the skin barrier recovery after injury. We also showed for the first time the expression of the tight-junction protein claudin-6 in human skin [8]. However, the relevance of this finding remained unknown until 2003 when Quan and Lu suggested that claudin-6 may function as a tumor suppressor at least in breast tissue [9].

In this context, we were prompted to investigate in depth the mechanisms of the protective effects of the TWFULB on human skin, on four grounds, i.e. 1) its antioxidant properties, 2) its ability to induce and/or reinforce skin endogenous antioxidant defence mechanisms, 3) its capability to protect nuclear DNA from the action of UV irradiations, and 4) its effect on the cutaneous expression of the candidate "tumor suppressor" claudin-6.

\section{Materials and Methods}

\subsection{Reagents and Materials}

Dulbecco's modified Eagle medium (DMEM), Fetal calf serum and antibiotics were purchased from Life Technologies (Saint Aubin, France). Keratinocytes growth medium (KGM) was purchased from Promocell (Heidelberg, Germany). Acetone came from VWR International (Fontenay-sous-Bois, France).

Mouse anti-human claudin-6 primary antibodies came from Santa Cruz Biotechnologies (Santa Cruz, CA., USA). Goat anti-mouse secondary antibody coupled to Alexa Fluor ${ }^{\circledR} 488$ was purchased from Life Technologies (Saint Aubin, France). Tissue-Tek ${ }^{\circledR}$ came from VWR International (Leuven, Belgium). Dapi came from Roche Diagnostic (Mannheim, Germany). Thermal water came from Uriage-les-Bains, France.

SOD and catalase activity assay kits, TBARS (ThioBarbituric Acid Reactives species) assay kit and MTT assay kit, were purchased from sigma Aldrich (Saint Quentin Fallavier, France). Comet assay kit came from Cell Biolabs Inc. (San Diego, USA). Claudin-6 ELISA assay kit came from Antibodies-Online (Atlanta, USA).

\subsection{Thermal water from Uriage-les-Bains: Ionic Composition and Osmolarity}

Mean ionic content per liter:

$\begin{array}{llll}\text { Sulfates } & 2860 \mathrm{mg} & \text { Potassium } & 45.5 \mathrm{mg} \\ \text { Chlorures } & 3500 \mathrm{mg} & \text { Silicon } & 42 \mathrm{mg} \\ \text { Sodium } & 2360 \mathrm{mg} & \text { Zinc } & 160 \mu \mathrm{g} \\ \text { Bicarbonates } & 390 \mathrm{mg} & \text { Manganese } & 154 \mu \mathrm{g} \\ \text { Calcium } & 600 \mathrm{mg} & \text { Copper } & 75 \mu \mathrm{g} \\ \text { Magnesium } & 125 \mathrm{mg} & \text { Iron } & 15 \mu \mathrm{g}\end{array}$

Osmolarity of TWFULB is $275+/-40$ mOsm. 


\subsection{Cosmetic Formulations Composition}

\section{1) Formulation containing distilled water}

Aqua (Water) - Cetearyl Isononanoate-Cetearyl Ethylhexanoate-Glycerin-Polymethyl Methacrylate-Dimethicon-PTFE-Acrylates/C10-30 Alkyl Acrylate Crosspolymer-Phenoxyethanol-Cetyl Alcohol-Steareth-2-Steareth21-Polyacrylate-13-Parfum (Fragrance)-Sodium hydroxide-polyisobutene-benzoic acid-o-cymen-5-ol-biosaccharide Gum-4-Taurine-Polysorbate 20.

\section{2) Formulation containing TWFULB}

Aqua (Water)-Thermal Water From Uriage-les-Bains-CetearylIsononanoate-Cetearyl Ethylhexanoate-GlycerinPolymethylMethacrylate-Dimethicone-PTFE-Acrylates/C10-30 Alkyl Acrylate Crosspolymer-PhenoxyethanolCetylAlcohol-Steareth-2-Steareth-21-Polyacrylate-13-Parfum (Fragrance)-Sodium Hydroxide-PolyisobuteneBenzoicAcid-o-Cymen-5-Ol-Biosaccharide Gum-4-Taurine-Polysorbate 20.

\subsection{Cell Cultures, Treatments and Irradiations}

\subsubsection{Fibroblasts}

Normal human dermal fibroblasts were cultured in DMEM with fetal calf serum (10\%) and antibiotics, in a humidified incubator under a $5 \% \mathrm{CO}_{2}, 95 \%$ air atmosphere.

When the cells reached confluency, they were incubated in Hank's Balanced Salt Solutions reconstituted with different ratios of desionized water and TWFULB (100\%/0\%; 75\%/25\%; 50\%/50\%; 25\%/75\%; 0\%/100\%). Cells were then incubated in the absence (control) or in the presence of a mix of hypoxanthine/xanthine oxidase (HX/XO: $0.25 \mathrm{mM} / 20 \mathrm{mU}$ ) for $45 \mathrm{~min}$. At the end of the incubation period, cell viability was assessed and TBARs were quantified in incubation media.

\subsubsection{Keratinocytes}

Normal human keratinocytes were cultured in KGM in a humidified incubator under a $5 \% \mathrm{CO}_{2}, 95 \%$ air atmosphere, for a $24 \mathrm{~h}$ period. They were then incubated in the absence (control) or in the presence of increasing doses of TWFULB (10\% and 30\%) for an additional $24 \mathrm{~h}$. At the end of this incubation period, cells were placed in Phosphate Buffer Saline (PBS) and irradiated or not (controls) by UVB $\left(60 \mathrm{~mJ} / \mathrm{cm}^{2}\right)$. After replacement of PBS by KGM completed or not with TWFULB, the cells were incubated for a $4 \mathrm{~h}$ incubation period. At the end of this new incubation period, cells were harvested and the degradation state of DNA was evaluated by a "Comet assay".

\subsubsection{Skin Explants}

Normal human skin explants were cultured in DMEM with fetal calf serum (5\%) and antibiotics, in a humidified incubator under a $5 \% \mathrm{CO}_{2}, 95 \%$ air atmosphere. After a $4 \mathrm{~h}$ incubation period, a cosmetic product, either formulated with de-ionized or TWFULB from Uriage-Les-Bains, was then topically applied on the explants $(20 \mu \mathrm{l}$ per explant). After a 24 incubation period, explants were placed in PBS and were irradiated by UVA (4 J/cm²) and UVB $\left(0.2 \mathrm{~J} / \mathrm{cm}^{2}\right)$. They were then re-treated by cosmetics products (20 $\mu \mathrm{l}$ per explant) and they were placed in DMEM with fetal calf serum (2\%) for a $24 \mathrm{~h}$ period. At the end of this incubation period, explants were frozen in liquid nitrogen before to study 1) the epidermal claudin-6 expression using immunohistochemical techniques and ELISA quantization, and 2) the total intracellular catalase activity.

\subsection{Cell Viability Assessment}

Cell viability was assessed using a MTT assay kit. The assay was realized according to the kit provider’s indications.

\subsection{TBARs Assay}

TBARs were quantified in cell incubation media by using a TBARs assay kit. The assay was realized according to the kit provider's indications.

\subsection{SOD Activity Assay}

SOD activity of various solutions containing or not TWFULB was assessed by using a SOD activity assay 
kit.The assay was realized according to the kit provider’s indications.

\subsection{Catalase Activity Assay}

Intracellular catalase activity of skin explants was quantified by using a catalase activity assay kit. The assay was realized according to the kit provider's indications.

\subsection{Comet Assay}

Comet assay was realized by using a comet assay kit, according to the kit provider's indications. Briefly, cells were mixed with molten agarose before application to the OxiSelect ${ }^{\mathrm{TM}}$ Comet Slide. These embedded cells are then treated with a lysis buffer and alkaline solution, which relaxes and denatures the DNA. Finally, the samples are electrophoresed in a horizontal chamber to separate intact DNA from damaged fragments. Following electrophoresis, the samples are dried, stained with a DNA dye, and visualized by epifluorescence microscopy. Under these conditions, the damaged DNA (containing cleavage and strand breaks) will migrate further than intact DNA and produce a "comet tail" shape. A dedicated software then permit to measure the tail extent moment.

\subsection{Claudin-6 ELISA Assay}

Claudin-6 was quantified by using a specific ELISA assay kit. The assay was realized according to the kit provider's indications.

\subsection{Immunohistochemical Study of Claudin-6 Expression}

Skin explants were embedded in Tissue-Tek. Frozen sections $(10 \mu \mathrm{m})$ were cut using a cryostat and fixed in cold acetone for $10 \mathrm{~min}$. Non-specific binding sites were saturated by using fetal calf serum at $0.1 \%(\mathrm{v} / \mathrm{v})$ and the skin slices were incubated for $1 \mathrm{~h}$ at room temperature with primary Claudin- 6 antibodies. The skin slices were rinsed with a phosphate buffered solution and incubated for $1 \mathrm{~h}$ at room temperature in the dark with secondary antibodies coupled to fluorochromes (Alexa Fluor ${ }^{\circledR} 488$ ).

At the same time, cell nuclei were labeled with DAPI following the procedure described below: DAPI (dilution: $1 / 100)$ was added to the secondary antibodies in a solution containing $1 \%(\mathrm{w} / \mathrm{v})$ of bovine serum albumin.

\subsection{Statistics}

Data are expressed as means \pm S.D. of experiments realized at least, in triplicates $(n=3)$. The statistical significances were assessed by one way analysis of variance (ANOVA) followed by Holm-Sidak'sor Student Newman Keuls' tests (as indicated).

\section{Results and Discussion}

As shown in Figure 1, TWFULB was able to significantly and dose-dependently increase the survival rate of human dermal fibroblasts submitted to an $\mathrm{HX} / \mathrm{XO}$ stress. This effect was detectable at the first tested concentration of TWFULB (25\%) and reached a maximum at the highest tested concentration (100\% of TWFULB). Interestingly, TWFULB was able in the same time to reduce the lipid peroxidation induced by the HX/XO stress. As shown in Figure 2, TBARs quantified in the incubation media were dramatically decreased in the presence of TWFULB. This effect is also statistically significant and dose-related, indicating that the protective effect of the water against $\mathrm{HX} / \mathrm{XO}$ stress is most likely due to its antioxidant properties.

In order to confirm this hypothesis and to complete our analysis of the antioxidant potential of the TWFULB, we then addressed the possible SOD-like activity of this water. As shown in Table 1, TWFULB shows itself a significant SOD-like activity $(1271+/-121 \mathrm{U} / \mathrm{l})$. Interestingly, this activity was enhanced in the presence of amino-acids (MEM reconstituted with TWFULB: $2004+/-241$ U/1). This last result, because of the composition of the TWFULB, could reveal the formation of "manganese-glycine-bicarbonates" complexes in the MEM reconstituted with TWFULB; in fact such complexes are known to exhibit significant SOD-like activity [10] (Table 1). This first part on our study clearly shows that TWFULB affords an effective protection of the cutaneous tissue against oxidative stress. This protection originates from the unique ionic composition of the TWFULB which is able to counteract oxidative stress by acting per se, or through the formation of "manganese- 


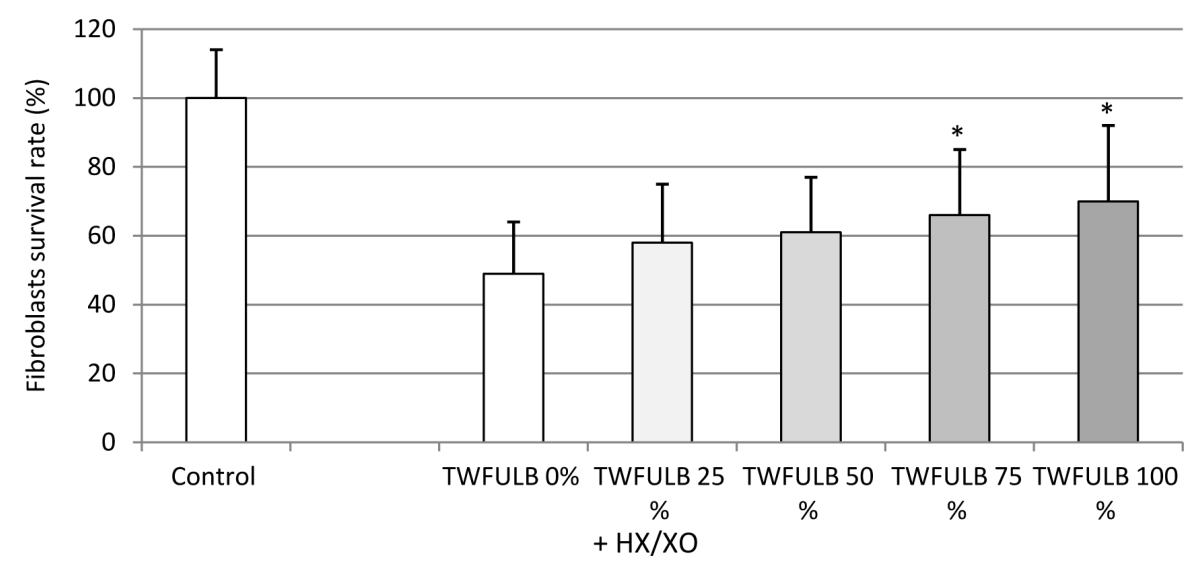

Figure 1. Effect of TWFULB on the survival rate of normal human dermal fibroblasts stressed with $\mathrm{HX} / \mathrm{XO}$. *: Significantly different from the "TWFULB $0 \%$ ” condition ( $\mathrm{p}<0.05$, one way anova analysis + Holm-Sidak's test); $\mathrm{n}=15$, from 3 independent experiments.

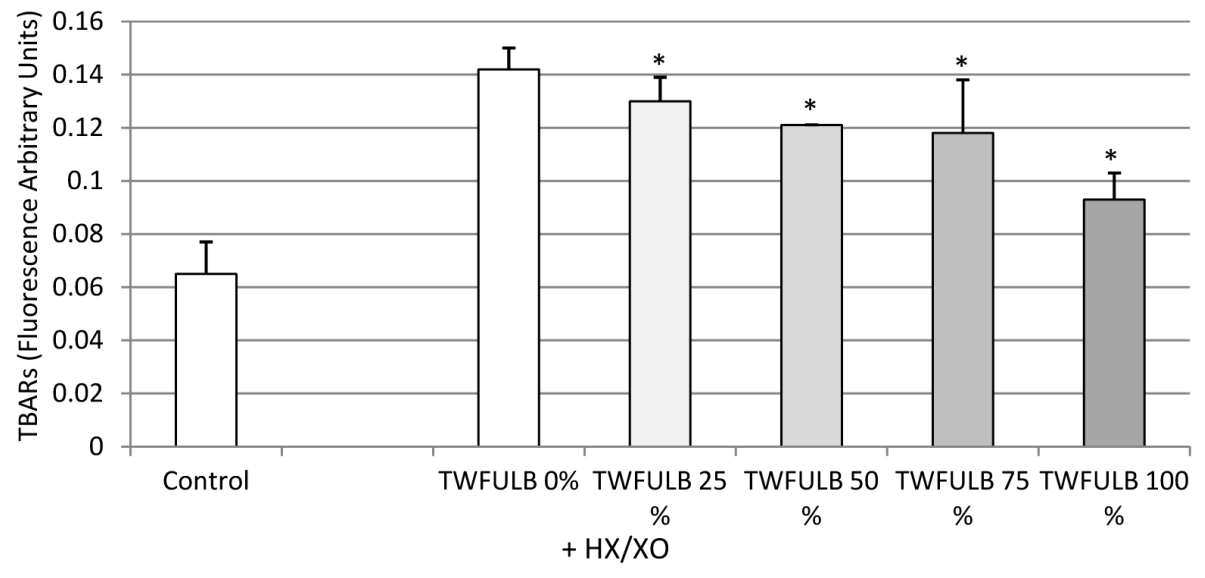

Figure 2. Effect of TWFULB on the lipid peroxydation in cultures of normal human dermal fibroblasts stressed with HX/XO. *: Significantly different from the "TWFULB 0\%" condition $(\mathrm{p}<0.05$, one way anova analysis + Holm-Sidak’s test); $\mathrm{n}=15$, from 3 independent experiments.

Table 1. SOD activity of different solutions containing or not TWFULB.

\begin{tabular}{cc}
\hline & SOD activity (U/l) \\
\hline Distilled Water & $332+/-27$ \\
TWFULB & $1271+/-141^{*}$ \\
MEM + Distilled Water & $507+/-49$ \\
MEM + TWFULB & $2004+/-178+$ \\
Manganese-glycine-Bicarbonates complexes $(50 \mu \mathrm{M}-50 \mathrm{mM}-23.5 \mathrm{mM})$ & $2958+/-317$
\end{tabular}

*: Significantly different from “Distilled Water” condition ( $\mathrm{p}<0.05$, one way anova analysis + Student Newman Keuls' test); +: Significantly different from “MEM + Distilled Water” condition ( $<<0.05$, one way anova analysis + Student Newman Keuls' test); $n$ $=6$, from two independent experiments.

glycine-bicarbonate" complexes. This last result could suggest additional indirect effects of the TWFULB which could be able to modulate intracellular pathways implicated in Reactive Oxygen Species (ROS) detoxification for example. Having shown the SOD-like activity of this water, we then extended and deepened our investigations by using a skin explant model that allows exploring at the cellular and molecular levels the response of the cell machinery to an UV stress. 
As shown in Figure 3, TWFULB was able to significantly and efficiently counteract the effect of UV irradiations on the intracellular catalase activity of skin explants. Indeed, as the UVA + UVB irradiations decreased the catalase activity in skin from $652.1 \mathrm{U} / \mathrm{mg}$ of proteins to $452.1 \mathrm{U} / \mathrm{mg}$ of proteins, a cosmetic formulation made with TWFULB allows the skin to partially recover its catalase activity: from $452.1 \mathrm{U} / \mathrm{mg}$ of proteins to 602.1 $\mathrm{U} / \mathrm{mg}$ of proteins. In this experiment, $75 \%$ of the UV irradiation effects were counterbalanced.

In the same experimental conditions, a cosmetic formulation made with distilled water did not restore the skin catalase activity which was in fact worsened to $358.2 \mathrm{U} / \mathrm{mg}$ of proteins.

This result is in line with the work of several authors showing that metals (notably zinc) are able to restore catalase activity in a model of gastrointestinal injured rats [11] or in zinc-deficient tomato plants [12] for example. The ionic composition of TWFULB which contain notably zinc, could one more time explain its efficacy to prevent loss of intracellular catalase activity in UV irradiated skin.

Regarding these TWFULB effects, we decided to go more deeply in the evaluation of its capabilities to protect cutaneous tissue and we studied the potential of our water to protect DNA from UV irradiations. We then resort to a comet assay, a method which quantitatively explores DNA damages at the global genome level (for a review, see [13]), in a normal human keratinocytes model irradiated or not by UVB.

As shown in Figure 4, the comet assay showed that $60 \mathrm{~mJ} / \mathrm{cm}^{2}$ of UVB lead to an increase in the mean of tail extent moments from $206+/-35$ (control) to $1524+/-59$, indicating a significant DNA degradation. In the same experimental conditions, TWFULB at $10 \%$ and $30 \%(\mathrm{v} / \mathrm{v})$ reduced the mean of the tail extent moments from $1524+/-59$ (UVB) to $1358+/-119$ and $1226+/-157$, respectively. We then really demonstrated a dose

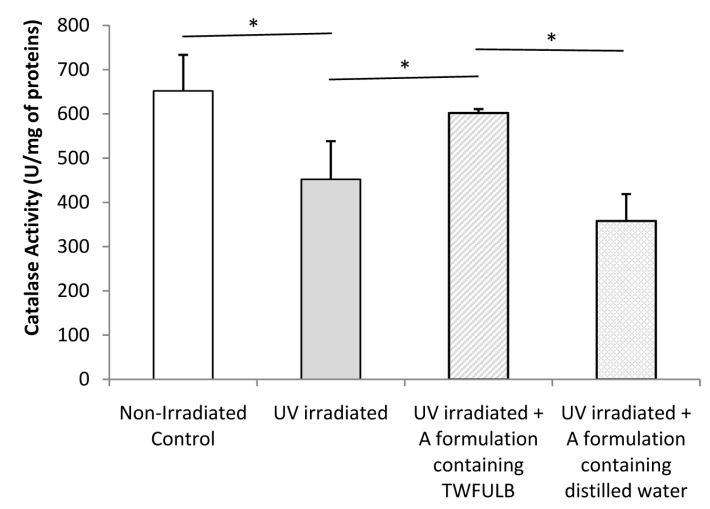

Figure 3. Catalase activity in skin explants irradiated or not by UVA + UVB and treated with formulations made with distilled water or with TWFULB. ${ }^{*}$ : Significant difference between the two conditions indicated by the bar $(\mathrm{p}<0.05$, one way anova analysis + Student Newman Keuls' test); $n=3$, from one experiment.

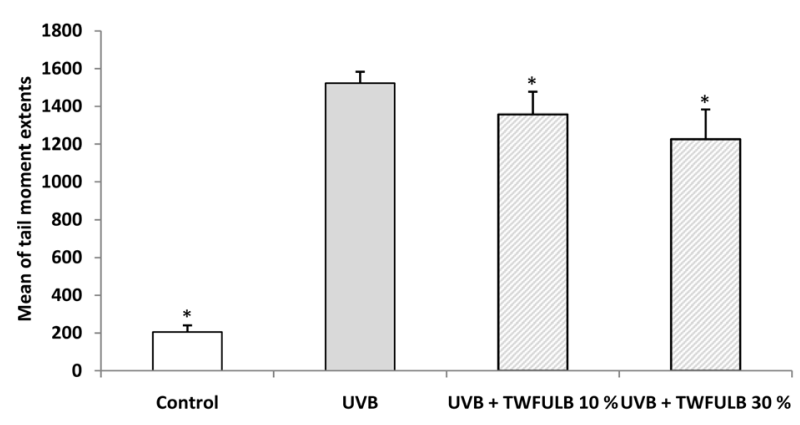

Figure 4. Comet assay realized with human keratinocytes irradiated or not by UVB and treated or not with TWFULB. *: Significantly different from the "UVB" condition ( $\mathrm{p}<0.05$, one way anova analysis + Holm-Sidak's test); 70 comet tails per condition were measured. 
related effect of TWFULB to reduce the UVB-induced overall DNA damage. This DNA protective effect of TWFULB against UVB irradiations is most likely due to its per se antioxidant effects and its ability to enhance the intracellular catalase activity.

As, 1) on the basis of the work of Quan and Lu, claudin-6 may function as a tumor suppressor, and 2) we previously showed claudin-6 expression in the skin [8], we were prompted to examine claudin-6 expression in our experimental conditions. Finally, UV irradiations are clearly involved in skin oncogenesis (for a review, see [14]), this was an additional incentive to clarify the effect of UV irradiations and TWFULB on claudin-6 expression in skin explants.

As shown in Figures 5 and 6, UVA + UVB irradiations led to a significant decrease in skin claudin-6 expression: from $1155 \mathrm{ng} / \mathrm{mg}$ of proteins in the control condition, to $912 \mathrm{ng} / \mathrm{mg}$ of proteins in the "UVs condition". In the same experiment we showed that a cosmetic product formulated with TWFULB applied at the skin surface before the UVs irradiation was able to counterbalance the UVs effect by restoring the claudin-6 expression from $912 \mathrm{ng} / \mathrm{mg}$ of proteins to $1070 \mathrm{ng} / \mathrm{mg}$ of proteins.

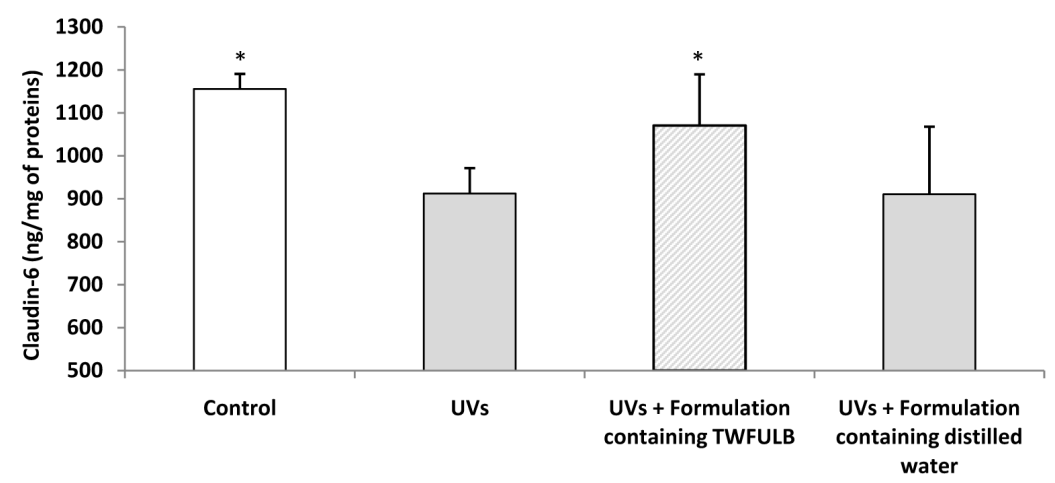

Figure 5. Claudin-6 expression in skin explants irradiated or not by UVA + UVB and treated with formulations made with distilled water or with TWFULB. * : Significantly different from the "UVs" condition ( $\mathrm{p}<0.05$, one way anova analysis + Student Newman Keuls’ test); $\mathrm{n}=3$, from one experiment.

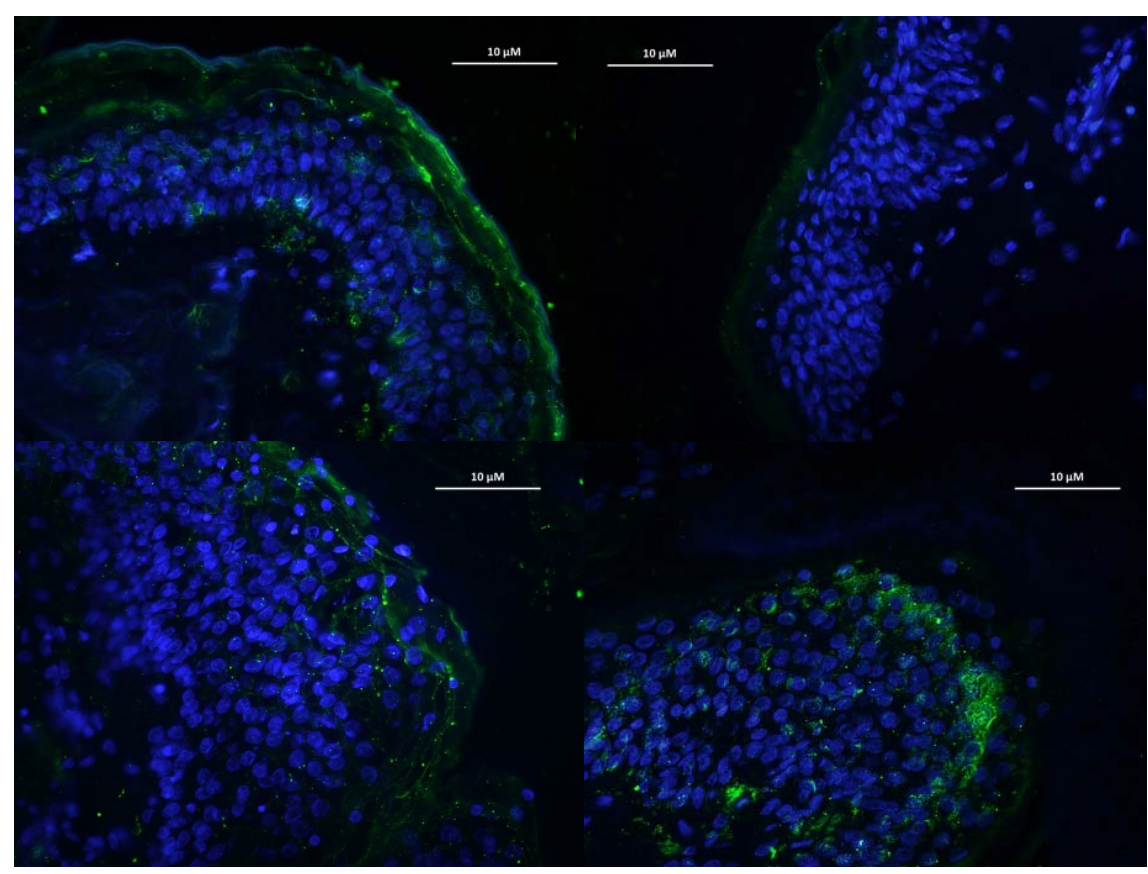

Figure 6. Claudin-6 expression in skin explants irradiated (B) or not (A) by UVA + UVB and treated with formulations made with TWFULB (C) or with distilled water (D). 
In the same experimental conditions, the application of a cosmetic product containing distilled water instead of TWFULB, did not led to any significant restoration of the skin claudin-6 expression (911 ng/mg of proteins).

\section{Conclusion}

In conclusion, here we validate a multiparametric investigation of the TWFULB skin protective effects. Indeed, we show that the thermal water from Uriage-les-Bains could afford a real protection to the skin in front of ROS and/or UVs stresses thanks to its direct and indirect antioxidant activities. TWFULB, acting through per se antioxidant activities and through the enhancement of intracellular antioxidant enzymes such as catalase, can counteract the UVB-induced DNA damages and could also help to fight against UVs-induced decrease of the putative tumor suppressor claudin-6.

\section{References}

[1] Braga, P.C., Ceci, C., Marabini, L. and Nappi, G. (2013) The Antioxidant Activity of Sulphurous Thermal Water Protects against Oxidative DNA Damage: A Comet Assay Investigation. Drug Research, 63, 198-202. http://dx.doi.org/10.1055/s-0033-1334894

[2] Braga, P.C., Dal Sasso, M., Culici, M., Spallino, A., Marabini, L., Bianchi, T. and Nappi, G. (2010) Effects of Sulphurous Water on Human Neutrophil Elastase Release. Therapeutic Advances in Respiratory Disease, 4, 333-340. http://dx.doi.org/10.1177/1753465810376783

[3] Braga, P.C., Sambataro, G., Dal Sasso, M., Culici, M., Alfieri, M. and Nappi, G. (2008) Antioxidant Effect of Sulphurous Thermal Water on Human Neutrophil Bursts: Chemiluminescence Evaluation. Respiration, 75, $193-201$. http://dx.doi.org/10.1159/000107976

[4] Merial-Kieny, C., Castex-Rizzi, N., Selas, B., Mery, S. and Guerrero, D. (2011) Avène Thermal Spring Water: An Active Component with Specific Properties. Journal of the European Academy of Dermatology and Venereology, 25, 2-5. http://dx.doi.org/10.1111/j.1468-3083.2010.03892.x

[5] Castex-Rizi, N., Charveron, M. and Merial-Kieny, C. (2011) Inhibition of TNF-Alpha Induced-Adhesion Molecules by Avène Thermal Spring Water in Human Endothelial Cells. Journal of the European Academy of Dermatology and Venereology, 25, 6-11. http://dx.doi.org/10.1111/j.1468-3083.2010.03893.x

[6] Seite, S. (2013) Thermal Waters as Cosmeceuticals: La Roche-Posay Thermal Spring Water Example. Clinical, Cosmetic and Investigational Dermatology, 6, 23-28. http://dx.doi.org/10.2147/CCID.S39082

[7] Lee, H.P., Choi, Y.J., Cho, K.A., Woo, S.Y., Yun, S.T., Lee, J.T., Kim, H.J., Lee, K.H. and Kim, J.W. (2012) Effect of Spa Spring Water on Cytokine Expression in Human Keratinocyte HaCaT Cells and on Differentiation of CD4(+) T Cells. Annals of Dermatology, 24, 324-336. http://dx.doi.org/10.5021/ad.2012.24.3.324

[8] Joly, F., Gardille, C., Barbieux, E. and Lefeuvre, L. (2012) Beneficial Effect of a Thermal Spring Water on the Skin Barrier Recovery after Injury: Evidence for Claudin-6 Expression in Human Skin. Journal of Cosmetics, Dermatological Sciences and Applications, 2, 273-276.

[9] Quan, C. and Lu, S.L. (2003) Identification of Genes Preferentially Expressed in Mammary Epithelial Cells of Copenhagen Rat Using Subtractive Hybridization and Microarrays. Carcinogenesis, 24, 1395-1599. http://dx.doi.org/10.1093/carcin/bgg129

[10] Varani, J., Ginsburg, I., Gibbs, D.F., Mukhopadhyay, P.S., Sulavik, C., Johnson, K.J., Weinberg, J.M., Ryan, U.S. and Waed, P.A. (1991) Hydrogen Peroxide-Induced Cell and Tissue Injury: Protective Effects of $\mathrm{Mn}^{2+}$. Inflammation, 15, 291-301. http://dx.doi.org/10.1007/BF00917314

[11] Ineu, R.P., Oliveira, C.S., Oliveira, V.A., Moraes-Silva, L., da Luz, S.C. and Pereira, M.E. (2013) Antioxidant Effect of Zinc Chloride against Ethanol-Induced Gastrointestinal Lesions in Rats. Food and Chemical Toxicology, 58, 522529. http://dx.doi.org/10.1016/j.fct.2013.05.022

[12] Wu, C. and Tsui, C. (1959) The Effect of Zinc on the Activities of Catalase, Peroxidase and Polyphenol Oxidase in the Tomato Plant. Acta Botanica Sinica, 8, 243-246.

[13] Collins, A.R., Oscoz, A.A., Brunborg, G., Gaivão, I., Giovannelli, L., Kruszewski, M., Smith, C.C. and Stetina, R. (2008) The Comet Assay: Topical Issues. Mutagenesis, 23, 143-151. http://dx.doi.org/10.1093/mutage/gem051

[14] Muller, H.K. and Woods, G.M. (2013) Ultraviolet Radiation Effects on the Proteome of Skin Cells. Advances in Experimental Medicine and Biology, 990, 111-119. http://dx.doi.org/10.1007/978-94-007-5896-4_8 